\title{
Perda dentária e seu significado na qualidade de vida de adultos usuários de serviço público de saúde bucal do Centro de Saúde Boa Vista, em Belo Horizonte
}

The loss of teeth and its meaning in the quality of life of adults who use the municipal oral health services of the Boa Vista Health Cen ter, in Belo Hori zonte

1 Faculdade de Odontologia da Un iversidade Federal de Minas Gerais.

Av. Antônio Ca rlos 6.627, Campus Pampulha, 31270-901,

Belo Horizon te MG.

vargasnt@task.com.br
Abstract The aim of this study was to find out the problems caused by the loss of teeth and by the lack of access to prosthetic treatment on daily life of adults who use the Municipal Health Services of the city of Belo Horizonte. The methodology used in this study was the qualitative research, acquired through open, semi-structu red interviews with 20 patients that went on a regular basis to the Boa Vista Health Center, a unit which belongs to the East County. The results showed that the problems mentioned by the patients were both functional and psychosocial, and the feelings rela ted to the loss of teeth were extremely nega tive. The patients pointed out the weaknesses of the adult oral health services, revealing its little effe $c$ tiveness because only the basic treatment is offered, leading to the extraction of teeth which could have been repai red and also to the unavaitability of prosthetic treatment for functional and appearance rehabilitation. It can be inferred that the Municipal Oral Health Program developed in Boa Vista Health Cen ter was insufficient to solve most of the dental problems that the adults have. Key words Teeth loss, Public policy, Quality of life
Resumo Es te trabalho objetivou estudar os problemas causados pela perda dentária e a falta de acesso à prótese na vida diária da população adulta, usuária de uma unidade de saúde da Secretaria Municipal de Saúde de Belo Horizonte (SMSBH). Trata-se de uma pesquisa qualitativa, realizada por meio de entrevistas abertas, semiestruturadas, com 20 pacientes atendidos no Centro de Saúde Boa Vista, pertencente à Regional Leste. Os re sultados mostraram que os problemas vivenciados pelos pacientes foram tanto funcionais quanto psicossociais, e os sentimentos relatados com a perda dentária, bastante nega tivos. Os pacientes apontaram as deficiências do serviço prestado ao adulto, revelando a sua pouca resolutividade, uma vez que só a atenção básica é ofertada, o que tem acarretado a extração de dentes em condições de serem recuperados. Tal situação é agravada pela não oferta da pró te sedentária para reabilitação estética e funcional. Concluiu-se que o Programa de Saúde Bucal da Secretaria Municipal de Saúde de Belo Horizonte, realizado no Centro de Saúde Boa Vista, mostrou-se insuficien te e ineficaz pa ra resolver a maioria das necessidades odontológicas que os usuários adultos apresentam.

Palavras-chave Perda dentária, Política pública, Qualidade de vida 


\section{Introdução}

Os estudos epidemiológicos clássicos, realizados na área de saúde bucal, têm fornecido informações sobre as condições bucais e as nece ssidades de tratamento das populações, apontando a cárie e a doença periodontal como as doenças mais prevalen tes na cavidade bucal, responsáveis pela maioria das perdas dentárias (Löe \& Brown, 1993). No en tan to, sabe-se que as condições sociais dos indivíduos bem como a prática odontológica hegemônica, que tem nas extrações dentárias a solução para o alívio da dor em populações de baixo nível socioeconômico, exercem um import a n te papel na qu e stão da perda dentária. No Brasil, a extração dentária em massa começa a partir dos 30 anos, como solução mais prática e econômica para os probl emas de saúde bucal (Pinto, 1997).

Bailit et al. (1987) estudaram as causas da mortalidade dental, con cluindo que a principal causa está associada à cárie e que a condição social é o outro fator importante. Nesse mesmo sen ti do, en con tra-se o trabalho de Guimarães \& Marcos (1996), que após examinar 414 pacientes, residentes em Belo Horizonte, ob s ervaram que praticamente $50 \%$ dos elementos dentais extraídos na classe social de baixa renda eram passíveis de rec u peração ou de con s ervação. Nesse mesmo trabalho, os autores concluíram que o número de dentes perdidos aumenta com a idade, sen do 2,5 ve zes maior na classe social de baixa renda do que na de alta, ficando cla ro o papel social na determinação da perda dentária.

Em usuários da rede pública de Belo Horizon te, o número de den tes ex traídos a partir da faixa etária de 25 anos aumenta muito, passando de 2,0 na faixa etária de 20 a 24 anos para 7,1 de 25 a 34 anos (Santos, 1996). Percebe-se, também, que a partir desta faixa etária as necessidades de tra ta m en to odontológico tornamse mais complexas, com gran de necessidade de tratamen tos en dodônticos, periodontais e de próteses. Os dados, obti dos por meio de um levantamento realizado em 1998 pela SMSBH entre seus usuários, mostraram que, no arco superi or, $32,5 \%$ dos paci en tes na faixa et á ria de 35 a 45 anos apresentavam necessidade de prótese parcial removível (PPR) e 21,1\% de prótese total (PTR); no arco inferior, 29,0\% tinham necessidade de PPR e $18,1 \%$ de PTR.

A Prefeitu ra Municipal de Belo Horizonte (PBH), através da SMSBH, modificou seu Programa de Sa úde Bucal, a partir de 1994, visan- do ampliar a população assistida e oferecer os s erviços para todas as faixas etárias, de acordo com os princípios do SUS (universalização, eqüidade e integralidade). Até aqu ele mom ento, a prioridade do serviço era apenas para as c rianças de 7 a 14 anos que estavam matriculadas nas escolas públicas. Para a viabilização desta proposta, a SMSBH lançou mão da oferta de atenção básica, acessível em todos os cen tros de saúde. Essa estratégia, preconizada pela OMS, visava a um salto qualitativo na saúde bucal dos indivíduos, porque, mediante a execução de proced im en tos de controle de infecção mais simples, permitia aumentar a cobertu ra da população.

No entanto, a atenção secundária, prevista nos princípios do SUS, e essencial para a formação de um programa de saúde bucal completo, ficou restrita a um atendimento cen trali$\mathrm{Z}$ ado (tratamen to en dodôntico e periodontal), de pouco acesso para os usuários, uma vez que a demanda é enorme e a cobertura muito pequena. Pa ra a população adulta, que geralm ente tem necessidades complexas de tratamento, a oferta exclu s iva de atenção básica tem causado um gran de número de ex trações dentárias.

Até recentemente, poucos estudos investigaram a relação en tre as condições bucais e seu impacto na vida das pessoas; mas, na última década, houve aumen to do interesse em quantificar as conseqüências das doenças. Vários instrumen tos foram de senvo lvi dos na ten ta tiva de conhecer e avaliar como os problemas bucais têm afet ado a vida diária das pessoas. Estes estudos têm sido dominados quase exclusivamente por modelos quantitativos utilizando questionários estruturados. Há, porém, muitas evidências, vindas das ciências sociais, mostran do que questionários estrutu rados expõ em mu i to pouco da variedade e profundidade dos sentimentos dos indivíduos. En trevistas abertas ou não estruturadas, por outro lado, proporci onam maior com preensão do comportamento humano e suas crenças. A ausência de respostas predeterminadas numa entrevista aberta oferece a possibilidade de perspectivas teoricamente novas de variáveis sociais e culturais, muitas ve zes não pensadas pelo pesquisador. Enquan to os questionários parecem querer testar hipóteses, as entrevistas abertas levantam hipóteses (Mac E n tee et al., 1997).

Minayo (1994) relata que um caminho possível para a construção do conhecimen to é a a preensão dos significados. Eles são fundamentais quando se deseja perceber a saúde e a doen- 
ça a partir de novos prismas, pois facilitam e ampliam a compreensão do processo saúde/ doença, o que pode re sultar em profundas implicações para a Saúde Pública. A busca desses significados, por meio da pesquisa qualitativa, pode ajudar a con s truir um conhecimen to que será de grande importância para a Epidemiologia, pois ampliará a compreensão de seu objeto, doen tes na população.

A com preensão dos probl emas diários, psicossociais e funcionais que as pessoas relatam por estarem desdentadas, e o que isso interfere na sua qualidade de vida, auxilia a interpretação do con hecimen to epidemiológi co já registrado na literatura sobre a quantidade de dentes perdidos na população adulta brasileira, a gran de nece s sid ade de pró tese e a falta de ace sso a esse serviço. Portanto, esse estu do utilizou a metodologia qualitativa para atingir seu objetivo de compreender o impacto que a perda den tá ria e a falta de acesso à pró tese causam na qualidade de vida das pessoas, e, ainda, avaliar, a través da visão do usuário, o serviço de saúde bucal ofertado à população adulta no Centro de Sa ú de Boa Vista da SMSBH.

Algumas teorias orientaram a análise desse trabalho. A prim ei ra delas foi sobre o significado do corpo e da boca. En ten de-se o corpo como um sistemabi oló gico que é afet ado pela religião, ocupação, gru po familiar, classe e outros in tervenientes sociais e culturais. É a cultura que dita normas para o corpo. A boca tem funções biológicas (mastigação) e sociais (falar, s orrir, comunicar), conota proximidade e intimidade nas relações sociais cotidianas e é instrumento de comunicação com o mundo e com a sociedade (Rod rigues, 1979).

A segunda teoria é sobre a aparência e estigma. Acredita-se que os sujeitos medem os outros pela aparência. $O$ rosto é a parte mais diferenciada do corpo. Aceitar a fisionomia é acolher o outro, dar-lhe aceitação (Barbotin, 1977). Portan to quan do um indiví duo tem um traço ou uma característica diferen te, uma deformidade física, pode ser rejeitado. Nesse caso, ele pos sui um estigma e está inabilitado para a aceitação social (Goffman, 1988). Portanto, a falta de dentes anteriores, na cultura ocidental, constitui uma desfiguração facial que configura um estigma.

$O$ con ceito de qualidade de vida que se ado tou nesse estudo foi o de Ruffino Netto (1992), que considera uma boa qualidade de vida aqu ela que oferece um mínimo de condições para que os individuos nela inseridos possam de- senvolver o máximo de suas potencialidades, sejam estas: viver, sentir ou amar, trabalhar produzindo bens ou serviços, fazendo ciência ou artes, vivendo pa ra ser meios utilitários ou utilitários fins (apenas enfeitando) ou simplesmente existindo. Entende-se, ainda, que a questão da qualidade de vida diz respei to ao padrão que a própria sociedade define e se mobiliza para conquistar, consciente ou inconscientemente, e, ao conjunto das políticas públicas e sociais que induzem e norteiam o desenvolvimen to humano, as mudanças positivas no modo e estilos de vida, cabendo parcela significativa da formulação e das responsabilidades ao denominado setor saúde (Min ayo et al., 2000).

A cavidade bucal, portanto, tem gran de influência na qualidade de vida tanto no nível biológico qua n to no psicológi co e social, a través da auto-estima, a uto-expressão, comunicação e estética facial (Gift \& Redford, 1992).

Sobre as políticas públicas de saúde, os princípios doutrinários do Sistema Único de Saúde (SUS), que são os da universalização, eqüidade e integralidade, foram os que norte aram as discussões de ste trabalho.

\section{Metodologia}

A metodologia utilizada nesse estu do foi a pesquisa qualitativa por meio de en trevistas abertas, semi-estruturadas. Os resultados foram produ zi dos pela análise de con te ú dodessas entrevistas. Toda a orientação metodológica foi baseada em Bardin (1977)). O Projeto de Pesquisa foi aprovado pelo Comitê de Ética em Pe s quisa da UFMG.

A amostra envo lveu pacien tes adul tos en tre 25 a 65 anos, aten didos no Cen tro de Sa ú de Boa Vista, situado na Regional Leste do município de Belo Hori zon te. O Centro de Sa ú de Boa Vista foi indicado pelos responsáveis técnicos do Di stri to por se tratar de uma das unidades odon tológicas on de eram realizadas mais exodontias.

Pa ra consti tuição da amostra foi estabel ecido um número ideal de 30 pacientes. O tamanho da amostra não é o fator determinante da significância do estu do qualitativo, que trabalha tipicamen te com amostras relativamen te pequenas, intencionalmen teseleci on adas (Sa ntos, 1999). A seleção desses indivíduos foi feita por meio das fichas clínicas do Cen tro de Sa úde Boa Vista entre os pacien tes que receberam alta do tratamento e que deveriam estar freqüentando, apenas, a manutenção preventiva. 
A faixa etária escolhida teve por base o trabalho de Santos (1996), que mostrou que o número de dentes extraídos aumenta muito a partir de 25 anos em Belo Hori zon te. As fich as foram examinadas uma a uma, obedecen do à ordem alfabética de arqu ivamento; aqueles pacien tes que estavam den tro da faixa etária, tinham dentes extraídos e necessitavam de prótese, eram listados com nome e en dereço. $\mathrm{Nu}$ ma primeira triagem, 50 pacientes foram selecionados, porque havia a possibilidade de que alguns não fos sem en con trados ou não quisessem participar do trabalho.

Os pacien tes passaram a ser procu rados seguindo a lógica da proximidade de endereço uns dos outros. Todos eram escl a reci dos quanto ao obj etivo do trabalho e assinavam o termo de con sentimento caso con cord as sem em participar. O paciente era con si derado não en contrado quando na primeira visita houvesse informações seguras da não existência da pessoa ou de sua mudança de en dereço; ou ainda se, após um número de três visitas ao local, não se conseguisse identificar a casa ou encontrar a pessoa. Todos os pacien tes foram en trevistados em sua pró priaresidência.

A coleta de dados foi realizada por meio de entrevistas semi-estruturadas, cujo rotei ro foi dividido em três unidades de con tex to: o Centro de Saúde, a perda dentária e saúde bucal. Em cada uma dessas unidades contextuais foram relacionadas questões que serviram de orientação para o de senvo lvimento das entrevistas, mas que não represen tavam um roteiro obrigatório, permitindo assim algum espaço para a participação do en trevistado na con dução e direcion amen to da conversa.

Ao iniciar a entrevista tinha-se como meta coletar dados sobre o indivíduo (idade, sexo, trabalho, tempo de moradia no bairro) e atingir certo relacionamen to e conhecim en to mútuo entre entrevistado e entrevistador. O interesse central da en trevista era representado pelos probl emas na vida diária das pessoas causados pela perda dental e a falta de acesso à prótese.

Foi realizado um pré-teste com cinco pacientes com o obj etivo de testar o rotei ro da entrevista. Após alguns pequenos ajustes, passouse a fazer as demais en trevistas, que foram gravadas em fita cassete e posteriormen te transcritas. O pesquisador utilizou um caderno de campo, no qual foram anotadas observações feitas du ran te a en trevista. Essas anotações era $m$ realizadas ao final, pa ra não con stra n ger os entrevistados.
Após a realização de 20 en trevistas, o pesquisador veri fi cou que já estava haven do muita repetição nos depoimentos. Con sidera n do que o material estava rico o suficiente para ser analisado, en cerrou a coleta de dados. Não foram en con trados nos endereços que con st avam no Cen tro de Sa ú de 14 indivíduos.

A análise de con teúdo pode envo lver tanto análise temá tica como textual. A análise textual implica examinar detalhadamen te os conteúdos léxicos e as estruturas sintáticas, e usualmen te toma a palavra como o el em en to básico a ser analisado. No presente trabalho optou-se pela análise temática, que se refere ao recon hecimen to de certos temas cen trais, ou idéias, no texto e ao enquadre em determinadas categorias. Os dados colet ados foram, port a n to, lidos exaustivamente, separados por temas e posteri ormente levantadas as categorias de análise.

\section{Resultados e discussão}

Foram en trevistados 20 pacientes, sen do 12 do s exo feminino e 8 do sexo masculino. A faixa etária variou de 28 a 65 anos, com 18 indivíduos casados, um solteiro e um divorciado. O tempo de moradia no bairro ficou entre 4 e 45 anos. Desses pacientes, 14 estavam trabalhando e 6 não estavam, sendo todos estes últimos do sexo feminino. Com relação ao grau de escolarid ade, 15 tinham o primeiro grau incom pleto, sendo que 10 des tes tinham cursado somen te até a quarta série; 2 tinham o primei ro grau completo; 1 tinha o segundo grau incompleto; e 2 tinham o segundo grau completo. A maioria morava em domicílio próprio (14 indivíduos); os demais alu gavam o imóvel. O rendimen to médio da população adstrita do Cen tro de Sa ú de Boa vista é de 3 salários mínimos.

Com relação à percepção do cen tro de saúde e do cirurgião-dentista, verifi cou-se que era positiva para a maioria dos usuários. Estes, porém, levantaram problemas com relação ao a tendimen to odontológico, médico e consideraram o acesso mui to difícil. Frases como: “O pessoal trata a gen te muito bem...", “...sempre tem esta fila..." "...é difícil conseguir fichas...” ilu stram os ach ados acima.

Belo Horizon te conta com 126 áreas de abrangência e 125 Unid ades de Saúde. A Organização Mundial de Saú de estabel ece a relação de uma Unidade de Saú de para 20.000 pessoas (Valla \& Siqueira, 1989). Segundo o IBGE (1996), a área de abrangência do Cen tro de Sa ú- 
de Boa Vista tem 8.125 habitantes, mas a população ainda precisa en frentar filas para o atendimen to e é po u co escl a recida qua n to ao acesso a alguns programas implementados. Isso demonstra a pouca participação do usuário no Centro de Saúde.

Seg un do a geren te do Cen tro, h o uve gra nde participação da população no momento de reivindicar a construção da unidade e o atendimento odontológico, com a formação do Conselho de Saúde Local. Após esse período, porém, h o uve dispersão do grupo. Percebe-se, no entanto, que a população não está satisfeita com o atendimento, critica o sistema, mas não se organiza para reivindicar mudanças e exigir sua participação. O envolvimento do usuário com o Cen tro é fundamental para que as propostas e programas sejam conhecidos e tenham os resultados esperados. No entanto, esse papel ativo que se espera de uma população cidadã, guardiã de seus direi tos individuais, ainda é pouco encon trado na maioria das comunidades brasileiras. A recu peração da cidadania depende do avanço de val ores democráticos, tais como igualdade, dignidade humana, participação, representativid ade, valores que foram abortados da população brasileira pelo Estado, du ran te o período da ditadu ra militar e que estão sen do recuperados lentamente. Somente a cidadania organizada pode cobrar um melhor desempenho dos serviços públicos (Sa n tos \& Ca rvalho, 1992).

Por outro lado, mesmo qu a n do se dá voz ao usuário através da representação nos Conselhos Municipais, Distritais ou Locais de Sa úde não se pode esperar que isto signifique uma participação efetiva da população na elaboração, com preensão e avaliação dos programas, já que ela é, politicamente, a parte mais fraca do sistema. Por essa razão, a maioria dos usuários nos conselhos de saúde tende a discutir o va rejo das secretarias de saúde, como o médico que atrasou, o sujeito que não obteve uma ficha, o mau atendimento feito por um funcionário. Essas são as questões que deixam os $u$ suá rios à von $t$ ade para falar e lhes dão a certeza de que estão participando e, também, porque se trata de uma ex pressão do mundo vivido, próximo daquilo que en $\mathrm{f}$ rentam no co tidiano. Con tudo, mesmo com essas limitações ainda encontradas na participação popular nos serviços de saúde, ela é extremamente importante para manter as insti tuições em funcion amen to (Sampaio, 1996).

A percepção do cirurgião-den tista foi sempre muito positiva: A dentista é muito boa, sé- ria. Faz um bom trabalho. A imagem do profissional parece sempre estar relacionada com a comunicação en tre ele e o paciente. As características do dentista ideal giram, principalmente, em torno de traços pessoais do profissional ligados à relação dentista-paciente (Gerbert, 1992). Apesar de ser considerada cuidadosa, calma e paci en te, o medo estava pre s en te: ...é mu ito paciente, o que foi bom para mim porque tenho muito medo. Isso se explica ten do em vista que as experiências anteriores dos indivíduos in flu enciam a sua posturaatual; se forem negativas, podem ser a causa desse medo (Rankin \& Harris, 1985). O medo do dentista é comum e está presente na grande maioria das pessoas.

Alguns pacien tes con si deraram o acesso ao tratamen to odon tológico muito difícil: "con seguir den tista é muito difícil...” e o atendimento limitado: “... den tista é mu i to limitado...”. “... tinha que ampliar o serviço." Tais ob servações se justificam uma vez que os procedimentos de atenção básica (restaurações plásticas, exodontias, cura tivos, raspagens e polimentos) são de baixa re solutivid ade para a necessidade de tratamen to dos adultos, que precisam, na maioria das vezes, de tratamentos endodônticos, periodontais, próteses unitárias, parciais removíveis e totais.

As causas da perda dentária mais comuns apontadas pelos pacientes foram a falta de informação e de condições financeiras para o tratamento e o tratamento inadequado recebido: Eu perdi meus den tes po rque não tinha condição, dinheiro pa ra tratar e aí começava a doer, corria lá e mandava arrancar. Ou ainda: Eu perdi meus den tes tinha só 16 anos (...) é aquela ignorância de pai falar assim: está doendo, a rranca e a gen te corria e arrancava. Esses achados con fi rmam os trabalhos de Pinto $(1997,2000)$ e Guimarães \& Marcos (1996), nos quais mostraram que o edentulismo, no Bra sil, tem causas sociais e que den tes passíveis de recuperação são extraídos por problemas econômicos. É extremamen te cruel que as pessoas sejam obri gadas a se mutilar por não possuírem condições econômicas para pagamento do tratamento necessário e por falta de acesso a um serviço públ i co que tenha uma resolutividade melhor e não ofereça somente extrações. Por precisarem de uma solução imediata para seus probl emas de dor de den tes, quepossibilite o retorno rápido a suas atividades cotidianas, os indivíduos aceitam a ex tração.

Nen huma correlação foi feita pelos usuários entre as doenças bucais e suas condições de vida. Apenas questionaram a prática dos 
dentistas que extraíam den tes por qualquer motivo. A esse respeito, não se pode esquecer que a prática odontológica dominanteexerce o papel de con tribuir para manter a força de trabalho em condições de produzir e de diminuir as tensões sociais, é extremamen te iatrogênica e mutiladora, e considera que os procedimentos preven tivos e restauradores não são importantes para a população carente (Bernd et al. , 1992).

Alguns pacientes demonstraram não ter aceitado a perda de seus den tes e exprimiram seus sentimentos com relação a essa situação: Quando perdi meus dentes chorei muito, achei que foi a maior tristeza da minha vida. Vários a utores en contraram rel a tos semelhantes (Valcanaia et al. , 1997; Fiske et al., 1995; Wolf, 1998). Os sentimentos com a perda dentária são mu ito negativos, os pacientes se sen tem apavorados, enver gonhados e têm a sensação de perd a irreparável. Na verdade, as pessoas não estão preparadas para a perda de seus den tes e não avaliam o impacto que este fato terá nas suas vidas.

Wolf (1998) explica que a erupção dentária é uma etapa mu i to importan te na organização psíquica dos indivíduos e por isso a sua perda pode implicar alterações com conseqüências para sua vida emocional. A autora chama a a tenção para as ex periências de cunho psíqu i co e para as ex periências cultu rais mais específicas que fazem com que a boca, os dentes, o sorriso e a mordida sejam referências pessoais e critério de aceitação social. Ela relata que em nossa or ganização sociocultural, a aparência é muito valorizada e quando as pessoas não alcançam o p adrão des ejado pela sociedade, elas se tornam vulnerá veis a sentimen tos de inferioridade, insegu rança e ver gonha.

Tomando a teoria de Rodrigues (1979) adotada nesse trabalho sobre o significado da boca, pode-se en ten der por que ela é tão importante para os indivíduos e por que qualquer problema que a envolva pode acarretar sentimentos tão negativos.

Os problemas na vida diária relacionados com a perda dentária foram de natu reza funcional como comer, mastigar ou falar; e social como mudanças no com portamen to, insatisfação com a aparência, prejuízo na aceitação social, dificuldade de acesso ao mercado de trabalho.

Relatos sobre a dificuldade de comer alimentos du ros e fibrosos causados pela perda dentária e a falta de prazer com a mastigação foram freqüen tes entre os en trevistados: “....atra- palha comer, minha boca está toda machucada”, “...não posso com er carne, n em tenho gosto mais." Parece que a habilidade para mastigar está relacionada com o número de dentes presentes e sua distribuição nas arcadas, aproximadamen te 20 den tes (Agerberg \& Carlsson, 1981). Wayl er \& Cha uncey (1983) relatam, no entanto, que embora não tenha sido provado que a mastigação seja realmen te necessária na digestão dos alimentos, alguns estu dos indicaram que os pacien tes com muitas perdas dentárias ou com próteses deficien tes tendem a selecionar alimen tos mais fáceis de en golir sem mastigar. Os autores acima não en contraram, porém, relação desta seleção de alimen tos com baixa nutrição e concluíram que a dieta modern a, cujo processamento se dá na cozinha ou na indústria, diminui a necessidade da mastigação. No entanto, os autores relatam que comer é uma função social importante, que uma quantidade de mastigação é necessária para a produção de saliva e su co gástrico e para facilitar a digestão. Masti gar e sen tir o go s to dos alimentos são essencialmente prazerosos e emocionalmen te necessários para o bem-estar do indivíduo e sua qualidade de vida (Ettinger, 1987).

Alguns pacien tes relataram ter problemas para falar por causa das falhas dentárias: ...estou falando e sinto que estou assobiando pelos lados da boca. A maioria dos trabalhos na literatura odon tológica mostra que os paci en tes passam a ter limitações funcionais com a perda dentária mas não especificam o problema com a fala. Apenas quando se usou o OHIP (Oral Health Impact Profile), um índice psicossocial de s envo lvido por Sl ade \& Spen cer (1993), verificou-se que parte dos pacientes tinha problemas para pronunciar palavras. Se é através da boca que o indivíduo se comunica com o mu ndo e é através dela que ele se expressa, pode-se imaginar o transtorno para uma pessoa que não con segue se fazer en ten der por não con seguir pronunciar corret a $\mathrm{m}$ en te as palavras.

A mudança no comportamentodos indivíduos devi do à perda dentária, no sen tido de escon der a boca, também foi relatada pelos entrevistados. Portanto, falar, comer e rir passaram a ser comportamentos evitados na frente de pessoas estranhas: ... todo mundo que fica sem dentes fica de certa maneira acanhado de co nversar, falar e so rrir, aauto - estima fica lá embaixo. Wolf (1998) explica essa mudança quando relata que os indivíduos dependem da aceitação social e, portanto, têm necessidade de 
acolhimen to por parte do seu gru po e o tem or de serem rejeitados pode interferir na sua forma de agir, sentir, pensar. A autora lem bra que o sorriso é um ritual de aproximação e é influenciado pela aparência dos dentes; a inibição do sorriso tem conseqüências, pois desconstróise a postura desejável, diminui-se a auto-estima, impede-se a demonstração da alegria e do acolhimento, prejudica-se o convívio social.

Por sua vez, a teoria de Goffman (1988) relata que o indiví duo com uma desfigu ração facial pode ser estigmatizado e, a partir daí, poderá experimentar relativamen te rápido uma mudança na personalidade aparente e passará a evitar os contatos com as pessoas normais, auto-isolando-se, torn a n do-se de s confiado, deprimido hostil, ansioso e confuso (considerase neste trabalho que essa desfiguração se configura pelo fato de a pessoa estar sem dentes). Essa mudança no com portamento pode ser explicada pelo receio de que seu defeito seja conhecido. Assim, fazem grandes esforços para passar despercebidos e começam a construir n ovas estratégias de adaptação.

Com relação à boa aparência, Wolf (1998) relata que ela é um fac i 1 i t ador para as trocas sociais, pois o indiví duo apren deu a avaliar-se segundo a apreciação dos outros. A sua imagem conta mais que a experiência, a habilidade, os afetos e o caráter, uma vez que será ju lgado, pelo menos inicialmente, pela visibilidade exteri or. O pri m ei ro olhar das pessoas é sem pre para o rosto, que está ligado à iden tid ade do indivíduo. A deformação desse rosto pode causar rejeição a essa pessoa e anular a atenção para os seus outros atri butos (Ba rbotin, 1977). O seguinte trecho reti rado das entrevistas revela isso: A gen te perde toda a beleza quando não tem dentes. A coisa mais importantesão os den tes da gente, a boca da gente é uma alegria.

Vários entrevistados revelaram ter problemas para con seguir trabalho por causa da falta de dentes anteriores: ...ficar sem den tes é duro, ainda mais na frente. Nem arrumar emprego a genteconsegue. Ou ainda, Eu trabalho de po rteiro noturno e quando fui arrumar esse emprego só dei xaram trabalhar à noite porque aí ninguém en x erga se eu tenho den te ou não. O corpo cumpre uma função ideológica e pode-se reconhecer no corpo das pessoas um dos diversos indic adores da posição social. Nesse sen tido, classificam-se as pessoas quan to à aparência, chegando, até mesmo, a habilitá-las ou não a determinados em pregos (Rodrigues, 1979). O autor explica que a beleza, vista sociologicamen- te, é, antes de mais nada, o produto de uma atividade orden adora e sistematizadora, a pon to de os indivíduos ficarem, de certa forma, moralmen te obrigados a pre servá-la. Não é difícil compreen der, portan to por que a aparência se torna critério importan te na seleção de em pregados que vão lidar com o públ i co.

Os problemas com os den tes anteri ores também foram vistos como impedimen to para uma boa recepção pelas outraspessoas: A boca é mu ito importante. É o cartão de visita da pessoa. (...) Pa ra as pessoas terem boa impressão da gen te é preciso ter os den tes bem cuidados. E ainda: A pessoa é bem re cebida se está com a boca legal. Quando está com cárie já fica aquele re ceio até de tomar no seu copo. Barbotin (1977) relata que acolher o outro significa aceitar sua fisionomia, assim como descartar um ro s to é recusar a convivência com aqu ela pessoa. O ros to do indiví duo está ligado à sua identidade. A sociedade espera que uma pe s s oa apareça para a outra mostra ndo correção na sua aparência, pois é através do seu ex terior que ela ex prime ou dem on stra como ela própria se vê e quer ser vista. No caso da sua aparência não estar adequada, segun doas normas da sua cultura, pode não ser bem recebida.

Com relação à próteseden tal, mu i tos en trevistados revelaram não usar ou improvisar alternativas por não possuírem condições financeiras para o pagamento: Não tenho prótese, não uso nada. É um probl ema. Não tenho condições financei ras de pagar. É caro. O programa de saúde bucal da SMSBH tem uma oferta de a tenção secundária (especializada) mui to re strita e não inclui a reposição dentária com a prótese, mesmo conhecen do a grande necessidade de prótese de seus usuá rios e o baixo nível socioeconômico dessas pessoas, como já foi levantado neste trabalho. Num cenário como este, percebe-se que a não oferta de prótese para a população não faz sentido, uma vez que esta deveria ser uma das pri orid ades diante do princípio da integralidade da atenção em saúde bucal.

A prótese apresentou-se, também, como solução para quem tem muitos problemas odontológi cos acumulados, com necessidade de intervenções em níveis de atenção básica e secundária e cujo tratam en to na rede privada ficaria bastante oneroso: Mas é muito difícil a gen te tratar, é mu i to caro. Meu irmão estava com a boca toda estragada, (...) fez um orçamento porque estava atrapalhando ele de arrumar emprego. Não deu conta de pagar (...) mandou arrancar todos os dentes porque era mais barato pôr dentadura. Hi storicamente, a prática odon- 
tológica tem realizado a ex tração dentária para eliminar um sintoma da doença, restaurandoa condição de normalidade (não sen tir dor), mas instaura a anormalidade (não ter dentes), que pode ser recon duzida à normalidade por meio de uma pró tese den tá riae, assim, o que é anormal passa a ser aceito como normal, social e mesmo profissionalmen te (Iyda, 1998). Bernd et al. (1992) também fazem considerações semelhantes, pois para tornarem os indivíduos aptos para o trabalho, muitas ve zes fazem extrações de dentes que poderiam ser recuperados. A ineficiência do setor público na solução dos probl emas bucais da população adulta fica evidente mais uma vez. É extremamente cruel as pessoas serem obrigadas a extrair seus dentes por não poderem pagar pelo tratam en tonecessário na sua recuperação. Está cla ro que é um equí voco dizer, quando som en te a atenção básica é oferecida para esta faixa etária, que exis te eqüidade no atendimento, uma vez que a resolutividade para esta população é mínima, e as extrações dentárias são, quase sempre, as únicas alternativas.
Finalmente, pode-se construir um modelo explicativo da perda dentária e suas conseqü ências para a vida dos indivíduos (Figura 1).

Assim, resumindo, a inexistência de um programa de saúde bucal eficien te na SMSBH, com atendimen to amplo nos três níveis de a tenção (integralidade), que não ofereça ao paciente apenas a atenção básica e que tra te os diferentes como diferentes, ou seja, tenha eqüid ade além da universalização do acesso, está levando o usuário adulto à perda dentária. Elementos dentais com possibilidades de recuperação são extraídos em função da estreita oferta de atenção secundária (integralidade). Por o utro lado, o paci en te des den t ado pelo sistema também não consegue a reposição dentária com a prótese, o que traria sua reabilitação f u nci onal e estética, uma vez que ela não é ofertada pelo serviço e ele não tem condições econômicas para fazê-la no sistema privado. Essa situação que está sen do viven ciada pelos usuários en trevis tados leva-os a ter diversos problemas na sua vida diária, com grande influência na sua qualidade de vida.

\section{Figura 1}

Modelo explicativo da perda dentária.

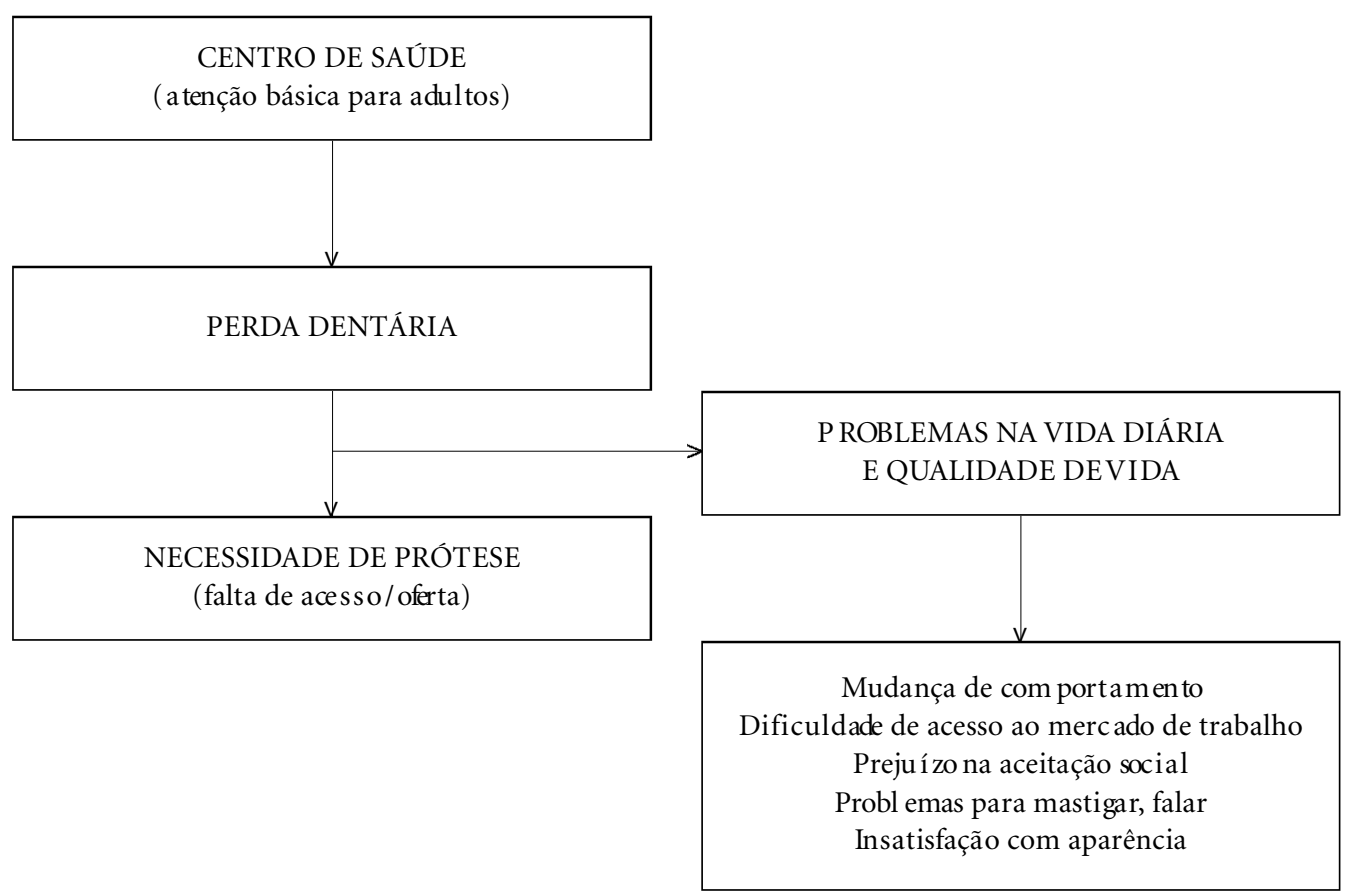


Em síntese, para tornar a saúde bucal um bem público, é necessário a participação da coletivid ade na implementação dos programas de saúde bucal coletiva, do engajamento e compromisso dos profissionais de saúde com a eficácia e eficiência desses programas e de todos os res pon sáveis pela gestão das ações e serviços de saúde, incluin do a seleção e uso das tecnologias nos diferentes níveis do SUS.

Segundo Mat tos (2001), quando se busca ori entar a organização dos serviços de saúde pelo princípio da integralidade, é preciso co$\mathrm{n}$ h ecer as percepções das nece s sid ades dos grupos e interrogar-se sobre as melhores formas de dar respostas a tais necessidades. O princípio da integralidade implica su perar reducionismos.

A esse re s pei to, é preciso salientar que a nova política naci onal de saúde bucal apresentada pelo Ministério da Saúde (Brasília, 2004) apon ta p a ra uma reor ganização da atenção em todos os níveis, visan doà promoção da qualid ade de vida. Tem como eixo de reori entação do modelo a linha do cuidado que traz con si go a proposta de humanização do processo. As novas diretrizes preten dem a ampliação e qualificação da atenção básica, asseguran do atendimen tos nos nívei s secundárioe terciário de modo a buscar a integralidade da atenção. Para ampliar a oferta de s erviços odon toló gi cos especializados, oMinisté rio repassará rec u rsos para a criação nos municípios dos Cen tros de Referência de Especialid ades Odontológicas que deverão estar sem pre in tegrados ao processo de planejamen to local. Esta nova proposta vem ao en con tro das necessidades e probl emas levantados pelos usuários nesse trabalho, poden do melhorar a qualidade dos serviços de saúde e humanizar suaspráticas.

\section{Con clusões}

O Programa de Sa úde Bucal da SMSBH, realizado no Cen tro de Saúde Boa Vista, centrado na atenção básica, mostrou-se insuficien te e ineficaz para resolver a maioria das necessidades odontológicas que os usuários adultos a presentam. A não oferta de atenção secundára pela rede pública faz com que muitos den tes que podem ser recu perados sejam extraídos.

As principais causas da perda dentária a pontadas pelos pacientes foram a falta de inform ação e condições econômicas para o tratamento. Os sentimentos relatados com a perda dentária foram bastante negativos, deixando cla ro que esses sentimentos não são decorrentes apenas dos problemas estéticos ou funcionais que a perda dentária traz, mas também porque os den tes e a boca têm um significado psicológico importante na formação do psiquismo humano. Os probl emas na vida diária relatados pelos usuários que perderam seus dentes foram de natureza funcional e social tais como: dificuldades para mastigar, falar, mudanças no comportamento, insatisfação com a aparência, prejuízo na aceitação social e dificuldade de acesso ao merc ado de trabalho, causan doforte impacto na qualidade de vida.

A reabilitação estética e funcional dos pacientes com a prótese mostrou-se difícil, uma vez que a rede pública de saúde não oferece esse serviço e por eles não possuírem condições econômicas de fazê-la na prática privada. $\mathrm{Pa}$ cien tes que já usam prótese revelaram ter dificuldades de ad a ptação e desconforto, mas continuavam usando-a em função da aparência.

Portanto, percebemos que os princípios doutrinários do SUS não estão sen do ob servados na estrutura organizacional, pois, nos espaços sociais, os serviços não são ofertados em todos os níveis de atenção. Essa deficiência provoca uma falta de prevenção na Incidência e um acúmulo na Prevalência das necessidades, com um increm en to constante em quantidade, complexidade, mutilação, insatisfação e seqü elas ao usuário do tratamen to pres tado em saúde bucal pelo Si s tema Único de Saúde.

\section{Colaboradores}

Este artigo foi extraído de tese de do utoramento da professora Andréa Ma ria Duarte Var gas na Escola de Veterinária da UFMG, área de Epidemiologia, c u jaorientadora foi a profe s s ora Helena Heloísa Paixão. 


\section{Referências bibliográficas}

Agerberg C \& Carls s onGE 1981. Ch ewingability in rel ation to dental and general health. Acta Odontologic. Scandinavian39(4):147-153

Bailit HL, Braun R \& Maryniuk GA 1987. Is peri odontal disease the primary cause of tooth extraction in adults? Jou rnal of Am erican Dental Association 114(1): 40-45.

Barbotin E 1977. El lenguaje del cuerpo. v. 2. Editora EUNSA, Pamplona.

Bardin L 1977. Análisedeconteúdo. Edições 70, Lisboa.

Bernd B, Souza CB, Lopes CB, Pi res Filho FM, Lisbôa IC, Curra LCD et al. 1992. Percepção popular sobre saúde bucal: o caso das gestan tes do Valão. Saúde em Deba te 34(3):33-39.

Brasil 2004. Política Nacional de Saúde Bucal. Mnistério da Saúde, Secretaria de Atenção à Saúde, Departamen to de Atenção básica, Coordenação Nacional de Saúde bucal. Centro de Documentação do Ministério da Sa ú de, Brasília.

Et ti n ger RL 1987. Oral disease and its effect on the quality of life. Gerodontics 3(4):103-106.

Fiske J, D avis DM \& Horrocks P 1995. A self-help group for complete den ture wearers. British Dental Jou rnal 178(5):18-22.

Gerbert B 1992. How dentists see themselves, their profession, the public. Jou rnal of American Dental As sociation 123(12):72-78.

Gift HC \& Red ford M 1992. Oral health and the quality of life. Clinic Geria tric Med i cine8(53):673-683.

Goffman E 1988. Estigma: notas sobre a manipulação da identidade deteriorada. Editora Guanabara Koogan, Rio de Jan eiro.

Guimarães MM \& Marcos B 1996. Expectativa de perda de dente em diferentes classes sociais. Revista do Conselho Regional de Odontologia de Minas Gerais 2(1): 16-20.

Insti tuto Brasileiro de Geografia e Estatística 1996. Contagempopulacional - 1996. In IBGE On-Line. Min is tério do Planejamento. Disponível em <www.ibge. gov. br> Ace s s ado em Jul. 2001.

Iyda M 1998. Saúde bucal: uma prática social, pp. 127-139. In C Botazzo \& SFT Freitas (orgs). Ciências sociais saúde bucal: questões e pers pectivas. Edusc, São Paulo.

Löe H \& Brown J 1993. Classification and epidemiology of periodontal diseases. Periodontollgy 2000 3(5):229238.

Mac En tee MI., Hole R \& Stolar E 1997. The significance of the mouth in old age. Social Science \& Medicine 45(9):1449-1458.

Mat tos RA 2001. Os sentidos da integralidade: algumas reflexões acerca de valores que merecem ser defen $\mathrm{d} i$ dos, pp. 39-64. In R Pinheiro, RA Mattos (orgs). O sentido da integralidade na atenção e no cuidado à saúde. Editora UERJ, IMS-Abra sco, Rio de Ja n ei ro.
Min ayo MCS 1994. Quantitativo equalit a tivoem indicadores de saúde: reven do con cei to s, pp. 25-33 In MFL Costa \& RP Souza (orgs.). Qualidade de vida: compromisso histórico da epidemiologia. Editora Coopmed, Belo Horizonte.

Min ayo MCS, Hartz ZNA \& Buss PM 2000. Qualidadede vida e saúde: um desafio necessário. Ciência e Saúde Col etiva 5(1):25-33.

Pinto VG 1997. Epidemiologia das doenças bucais no Brasil, pp. 27-41 In L Krieger. Promoção de saúde bucal. Ed. Artes Médicas, São Paulo.

Pin to VG 2000. Saúde bucal coletiva. Ed. Santos, São Paulo.

Rankin JA \& Harris MB 1985. Dental anxiety: the patien t's point of vi ew. Journal of American Dental Association 109(1):43-47.

Rod rigues JC 1979. O tabu do corpo. Ed. Adhiamé, Rio de Ja neiro.

Ruffino Net to A 1992. Qualidade de vida: com promisso histórico da Epidemiologia. Saúde em Deba te 35:6367.

Sampaio LFR 1996. A participação popular nos con s elh os de saúde, pp. 37-41. In LFR Sampaio et al. (orgs.). Os a li cerces da utopia: saúde e cidadania no SUS de Brumadinho. Ed. Prefei tu ra Municipal, Brumadinho.

Sa n tos I \& Carvalho GI 1992. Das formas de con trole social sobre as ações e os serviços de saúde. Saúde em Deba te 34(3):60-92

Santos RM 1996. Distribuição das doencas da cavidade bucal nos usuários do Sis tema Único de Saúde de Belo Ho rizonte: Um estudo de prevalência. Dissertação de mestrado. Faculdade de Odontologia da Universi$\mathrm{d}$ ade Federal de Minas Gerais.

Santos SR1999. Métodos qualitatios e quantitativos na pesquisa biomédica. Jornal de Pediatria 75(6):401406.

Sl ade GD \& Spen cer AJ 1993. Development and evaluation of the oral health impact profile. Community Dental Health 11(3):3-11.

Valla VV \& Siqueira SAV 1989. O Centro Municipal de Saúde e a participação popular, pp. 91-115. In NR Costa, MCS Minayo, CL Ramos \& EM Stotz. Demandas populares, políticas públicas e saúde. Editora Vozes, Petrópolis.

Valcanaia TC, Souza Filho A \& Ramos Filho S 1997. Melhoria na qualidade de vida de paci en tes com uso de próteses implanto-suportadas. Revista Brasileira de Cirurgia e Implantodontia 4(2):53-63.

Wayler AH \& Chauncey HH 1983. Impact of complete den tu res and impairred natu ral dentition on mastigatory perform anceand food choice in health aging men. Journal Prostetic Den tistry 32(49):427-433

Wolf SMR1998. O significado psicológico da perda do s dentes em sujeitos adultos. Revista da Associação Paulista de Ciru rgiões Den tistas 52(4):307-316.

Artigo apresen t ado em 4/10/2004

Aprovado em 26/11/2004

Versão final apresen tada em 10/12/2004 